\title{
Understanding Young Adult's Adjusting to Diabetes Management Behavior: Multi-Methods Approach
}

\section{Willemse M, Deacon E* and Segal D \\ North-West University, South Africa}

*Corresponding author: Dr Elmari Deacon, Optentia Research Focus Area, NorthWest University, PO Box 1174, Vanderbijlpark, 1900, South Africa, Tel: +27 72480 7901; Email: elmari.deacon@nwu.ac.za

\section{Research Article \\ Volume 3 Issue 7}

Received Date: July 27, 2018

Published Date: August 20, 2018

\section{Abstract}

Background: Young adults living with diabetes experience unique challenges related to their developmental stage. A number of variables, including illness perception, social support and meaning in life influence their adjusting to diabetes management behaviour. Although managing diabetes is a challenge to most young adults, this study proposed studying those who does manage diabetes well to get a better understanding of the processes involved.

Objective: The aim of this study was to explore and understand how developmentally appropriate challenges and psychological health variables play a role in diabetes self-management of young adults living with well-controlled type 1 diabetes in South Africa.

Design: This study employed an exploratory, multi-methods design with an overall qualitative, quantitative (QUAL quant) research approach.

Setting: Specialised centres for diabetes care were used.

Participants: Six (6) young adults, aged 19 - 25 years, with well-controlled type 1 diabetes took part in this study.

Methods: Semi-structured interviews were audio-recorded, transcribed, and thematically analysed. Data were coded using ATLAS.ti. Through an iterative process, major themes emerged from the data. Self-report questionnaires were used to quantify inter-relationships in thematic data.

Results: Diabetes management behaviours were found to be compromised by the young adult's challenges towards establishing an autonomous identity, experiences of emotional distress as well as frustration pertaining to diabetes treatment plans and experience of social support received by family and friends.

Conclusions: Seeking a new normal lifestyle incorporating diabetes management behaviours was the most significant contributor to engage in a meaning-making process.

Keywords: Young adults; Diabetes management behaviours; Personal model; Meaning; Social support; Multi-methods research 


\section{Psychology \& Psychological Research International Journal}

\section{Contribution of the paper}

Young adults living with type 1 diabetes experiences emotional distress related to the management of diabetes resulting in poor adherence to treatment plans. The results of this study emphasized that young adults should be guided towards creating a new normal in living with diabetes in order for them to manage their diabetes well.

\section{Introduction}

Young adulthood, the years from 18 to 25 is a time of dramatic change, accomplishments, and risk taking [1]. Every young adult faces normal development-stage challenges such as moving out of the parents' home and establishing a sense of autonomy. Among young adults living with type 1 diabetes, these challenges may be compounded by diabetes-related self-management needs $[2,3]$.

The young adult's psychological well-being is said to be influenced by the manner in which they cope with the developmental stage challenges [4].Considerable research have been done on emphasising the importance of experiencing this development stage in a positive way, as to limit psychological manifestations such as anxiety, increased dependence, passivity and poor self-image $[5,6,7,8]$. Research highlighted that the psychosocial and cognitive resources of the young adult, as well as the availability of social support, determined the successful management of the challenges created throughout the journey towards adulthood [9] and their experiences of psychological well-being [10].

However, for the young adult living with type 1 diabetes, the journey towards adulthood is slightly more engaging as this individual not only needs to adapt to all the developmental challenges and stage-generated changes, but also needs to deal with purposeful diabetesrelated challenges [9]. Diabetes-related challenges include: (1) the diagnosis, which can be experienced as traumatic; (2) commencing with the diabetes care plan, which must be followed very meticulously; (3) attending diabetes-specific educational sessions that allow for the successful integration of diabetes into the lifestyle of the individual; and, (4) the reality of developing diabetesrelated complications $[9,11,12]$. In addition, young adults with type 1 diabetes reported higher levels of emotional distress, hormonal changes, and autonomy challenges as compared to their age group without type 1 diabetes [13] and that could negatively influence their diabetes selfmanagement behaviours.
There is little doubt that type 1 diabetes can be a tough condition to live with, not to mention the impact on the quality of life that can be great. The young adults living with type 1 diabetes face endless demands and challenges in order to achieve glycaemic control targets as recommended by medical professionals [14]. It is argued that positive experiences during this development stage allow young adults with type 1 diabetes, to spontaneously enter into new diabetes teams, discussions on how to integrate diabetes with a new, "grown-up" lifestyle and how to integrate diabetes into their sense of self or identity $[11,12,15]$. It is also well researched that young adults require support from family, friends, and physicians in order to cope and transit to healthy, productive, and diabetes-responsible individuals, taking self-care seriously [16].

Self-care for the advancement of well-being is a cornerstone in the management of diabetes [17]. Young adults living with type 1 diabetes need to make sound decisions when self-managing their condition. Diabetes management decisions need to be aligned with the recommended diabetes care plan as set out by physicians. This is a stereotyped view, which neglects patient autonomy and is one of the major reasons that outcomes, such as adherence to the diabetes care plan and HbA1c levels are poor. A treatment plan that is not derived from a shared decision making process is doomed to failure [18]. Research confirmed, the most recommended successful approach is one based on shared decision making and respect for patient autonomy, acceptance of the condition (including the ups and downs) and finding an individualised strategy to manage the diabetes adversities $[5,6,7,8]$.

If young adults living with type 1 diabetes are expected to adhere to the diabetes care plan, they must acquire an understanding of diabetes that is flexible, coordinated and interconnected with every other aspect of their lives [1]. Research indicated an association between the young adult's perception of diabetes (identified as the personal model of diabetes), diabetes self-management behaviours, clinical outcomes (blood glucose levels or HbA1c), and meaning created from live events and psychological well-being $[19,20,21,22,23]$. Those who studied the psychological well-being of young adults living with type 1 diabetes argued that psychological wellbeing is corroborated by the level of social support received [24]. In addition, adjustment to the demands of type 1 diabetes is argued to be better in those who are open to new information, are engaging, and use critical thinking skills in enhancing their self-efficacy [25]. 


\section{Psychology \& Psychological Research International Journal}

Young adults experience diabetes self-management behaviours as stressful, which makes it harder to adhere to the diabetes treatment plan [26]. Previous studies have reported that young adults experienced the following when adopting and maintaining diabetes selfmanagement behaviours: (1) identity development was challenging; (2) intense emotional distress went along with diabetes self-management behaviours; and (3) diverse experiences of the diabetes treatment plan and social support received from friends and family [27]. However, less is known about how developmentally appropriate challenges and psychological health variables play a role in diabetes self-management. In the present study, the purpose was to explore and understand this relationship by investigating young adults living with type 1 diabetes in South Africa.

\section{Materials and Methods}

\section{Research Design}

This study employed an overall qualitative, quantitative (QUAL - quant) research approach. This approach was embedded within an exploratory, multimethods design. A critical realism framework with concurrent triangulation approach was chosen to form a deeper understanding of the diabetes self-management behaviours employed by young adults living with type 1 diabetes $[28,29]$. In adopting a critical realist stance, the contribution of both scientific and objective knowledge are recognized. This implies that an individual's experiences and meanings can coexist and affect behaviours as well as wider social structures [30].

\section{Research Setting}

The research was conducted at the Centre for Diabetes and Endocrinology (CDE), in the Gauteng Province, South Africa. Data collection took place between October 2015 and June 2016.

\section{Ethical considerations}

Permission to carry out the study was obtained from the Humanities and Health Research Ethics Committee (HHREC) of the Faculty of Humanities of the North-West University (NWU-HS-2015-0156). Independent, written, informed consent were received from all participants in this study. Participation was voluntary and interviews were conducted in either Afrikaans or English, audiorecorded, and transcribed verbatim. Questionnaires were only available in English.

\section{Participants}

Purposive sampling resulted in 6 young adults participating in this study. Biographic distribution was as follows: four females (67\%) and two males (33\%). The average $\mathrm{HbA} 1 \mathrm{c}$ levels at the time of the study was $7.3 \%$ (45 mmol/mol (recommended range 6.3\% - 8.0\%; 39 $\mathrm{mmol} / \mathrm{mol}-49 \mathrm{mmol} / \mathrm{mol}$ )). The mean age was 21.3 years (range $19-25$ ) at the time of the study. The mean time since diagnosis of diabetes was 12 years (range 2 22). Out of the six participants, five (83\%) were European and one (16\%) was of Cape Malay decent. None of the participants reported significant co-morbidity that could have influenced their health beliefs or coping responses. The following steps - data collection, data analysis, data validation/legitimation, and data interpretation - were interactive and cyclical steps in the multi-methods research process.

\section{Data Collection}

Participants within the age range of 18 - 25 years, were invited to participate in this study. Screening took place to ensure the inclusion criteria was met. This included that participants must have been diagnosed with type 1 diabetes, and experience well-controlled HbA1C levels $(<8 \%, 64 \mathrm{mmol} / \mathrm{mol})$. The researchers acknowledge that international diabetes bodies, and the Society for Endocrinology, Metabolism and Diabetes of South Africa (SEMDSA) recommend that adults should have a target $\mathrm{HbA1c}$ of $<7 \%$ (53 $\mathrm{mmol} / \mathrm{mol}$ ) to be considered well-controlled. This study, however, used an $\mathrm{HbA1c}$ level that was the average for a year, and since fluctuations in HbA1c do normally occur over a year, this level captured most candidates. Hence, it could be viewed as a valid measure of adoption and maintenance of effective diabetes behaviours. Participants had to be fluent in English and could not suffer from any other condition that may influence their diabetes management behaviours. Potential participants were invited to take part in the study via advertisement. All participants that were approached, agreed to participate and provided written, informed consent. There were no refusals to participate or any drop-outs once research began.

The interviews were conducted by a female researcher with experience in interviewing. She had no prior relationship with the participants. At the time of the study, she was employed as a part-time lecturer and registered psychologist. The researcher kept field notes of interviews and incorporated it with data analysis. No repeat interviews were necessary. Interviews lasted between 20 and 60 minutes. The qualitative data were 
collected through face-to-face, semi-structured interviews conducted at the CDE. A flexible interview schedule was compiled, covering the basic contribution themes to be enclosed [31]. Interview questions and or prompts, such as (1) Explain how your life changed after being diagnosed with diabetes; (2) How do you manage your diabetes?; (3) How does your understanding of diabetes influence your management of the condition?; (4) Explain how social support influence your management behaviour and (5) How do you make meaning of your diabetes management behaviours? were used during the interviews. A brief, verbal summary of the project was given prior to obtaining the written informed consent. Permission to audiotape each interview was obtained individually and was used as the opportunity to establish positive rapport with the participant. For the purpose of this study, questionnaires covering the psychosocial variables in questions were incorporated. The questionnaires included were:

The Diabetes Self-Management Profile - Self report (DSMP-SR) [32]: This 24-item self-report instrument (not divided into subscales) quantifies diabetes self-care management behaviours over the preceding three months. The question-format changes throughout the instrument and participants indicate the most applicable answer to each question. Item examples are: (1) If you think you are having a low blood sugar, how often do you check your blood sugar before treating?; (2) In the past 3 months, before you ate more than usual, did you make any insulin changes?;(3) In the past 3 months, if you had two blood sugar results above 13 in a row, how often did you do a ketone test? The DSMP-SR reported a Cronbach alpha coefficient of 0.82 [32,33].

The Pemberton Happiness Index (PHI) [34]: The PHI produces a combined index of an individual's wellbeing that is the sum of different subscales. Subscales include 11 items on remembered well-being (general, meaning, psychological well-being, and social well-being) and 10 items on experienced well-being (i.e. positive and negative emotional events that have possibly taken place the day before). Each item is rated on a 10-point Likert scale from 0 (fully disagree) to 10 (fully agree). Examples of items on remembered well-being are: (1) I am very satisfied with my life; (2) I enjoy a lot of little things every day; and (3) I have a lot of bad moments in my daily life. Experienced well-being items are: (1) Something I did made me proud; (2) I gave myself a treat; and (3) I was bored for a lot of the time. The PHI reported a Cronbach alpha coefficient of 0.82.-0.83 [34].
The Revised Illness Perception Questionnaire (IPQ-R) [35]: This 70-item, self-report instrument identifies the five subscales within the representation of an illness. These subscales are: (1) identity - describes 14 symptoms associated with the illness. Participants decide whether or not they have experienced those symptoms and if they perceived those symptoms to be related to their illness. The participants respond by either circling "yes" or "no" to each question. Examples of symptoms are; pain; sore throat; fatigue and sleep difficulties; (2) consequences - the expected long and short term outcome of the illness; (3) causes - ideas on how the illness was contracted or what caused the illness; (4) timeline - beliefs concerning how long the illness will last; and (5) cure and control - beliefs on whether or not the individual can recover from or manage the condition $[36,37]$.

Responses to the other four subscales are on a 5-point Likert scale, ranging from 1 (strongly disagree) to 5 (strongly agree). Examples of items are: (1) My diabetes will last a short time; (2) My treatment can control my diabetes; (3) I do not understand my diabetes; (4) I get depressed when I think about my diabetes; and (5) My diabetes may have been caused by chance or bad luck. The IPQ-R reported a Cronbach alpha coefficient of 0.75-0.89 for the IPQ-R complete scale [35].

The Meaning in Life Questionnaire (MLQ) [38]: This instrument consists of 10 items on a 7-point Likert scale ranging from 1 (absolutely untrue) to 7 (absolutely true). It is divided into two subscales, namely: (1) presence of meaning; and (2) search for meaning. Items on the presence of meaning subscale are concerned with the degree to which the individual feels that his/her life already has some source of meaning (e.g. (1) I understand my life's meaning; and (2) My life has a clear sense of purpose). The search for meaning subscale examines the extent to which an individual is searching for meaning in his/her life (e.g. (1) I am always looking to find life's purpose; and (2) I am searching for meaning in my life). Steger [39] reported that there were no formal cut-off scores to determine what constitutes a high score or a low score on the MLQ.

\section{The Modified Diabetes Social Support} Questionnaire (M-DSSQ) [40]: This instrument includes two subsections, namely, support from family (58 items) and support from friends (28 items) on a 5point Likert scale ranging from 0 (never) to 5 (at least once a day). Participants also complete a subscale on how he/she feels about the support behaviour mentioned in 


\section{Psychology \& Psychological Research International Journal}

each item, also on a 5-point Likert scale ranging from 1 (unhelpful or not supportive) to 3 (very supportive). The family support subscale consists of five subscales, namely, (1) guidance and supervision; (2) encouragement of selfcare and exercise; (3) support in critical situations; (4) nourishment; and (5) emotional support. The friendssupport subscale consists of five subscales, namely, (1) guidance and encouragement; (2) help in critical situations; (3) nourishment; (4) empathy; and (5) help in exercise. Examples of items are: (1) Give you insulin; (2) Nag you until you do your testing; (3) Do the grocery shopping for your meals; (4) Remind you to exercise; and (5) Give you things to read on diabetes care. Both the MDSSQ-family and M-DSSQ-friends reported Cronbach alpha coefficients of 0.76-0.92 [40,41].

\section{Data Analysis}

For the purpose of this study, the qualitative and quantitative data were combined and data integration took place. A coherent whole, i.e. meta-inference, [42] was formed [43].

Interviews were read thoroughly, transcribed, and a thematic analysis was conducted according to guidelines published by Braun and Clarke [45]. The transcripts were entered in ATLAS.ti ${ }^{\mathrm{TM}}$ software (Version 7) [44] and the analysis was iterative throughout, allowing for the emergence of patterns in the data $[45,47]$. Patterns in the interviews were coded, compared and contrasted between and within interview transcripts, using constant comparative methods [48]. Interview codes were organised into themes reflecting the research question. No new contributing themes emerged after interview 3, therefore, data saturation had taken place.

All transcripts were coded by the researcher and a cocoder, an independent third party with outstanding subject knowledge, as well as a panel of experts in the field of diabetes. This ensured reliability of the study [45,46]. Emergent contributions were compared and discussed between coders at regular meetings until consensus on key issues was reached. A qualitative data analysis software package (ATLAS ti ${ }^{\mathrm{TM}}$ version 7) [44], was used to facilitate data coding and ease the retrieval of data.

For the purpose of this study, the questionnaires were hand-scored by the researcher. The results of the quantitative data were used to verify and enhance findings emerging from the qualitative data. Integration of the qualitative data with the quantitative data took place during the interpretation phase of the study [28].

\section{Rigor}

Throughout this study, attention was given to ensure rigorous research was done. During the qualitative stage, the evidence-based guidelines for rigor in multi-methods research [49] was followed. These guidelines included: (1) the research formulation stage - it was clearly stipulated in both the conduct and reporting how the different research components interacted with each other; (2) the research planning stage - the sampling design and overall research design have been comprehensively accounted for; (3) the research implementation stage - the data collection, data analysis, data legitimisation, and interpretation generated by the researcher fit the data well; and (4) the research dissemination stage - during the course of the study, discussions with knowledgeable colleagues were conducted in order to ensure credibility of findings, and sensitivity was communicated pertaining to the participant's realities and the negotiated realities. Regardless of the specific tool or research method used, the key was to provide the reader with a clear audit trail, as well as well-considered and justified rationales for the decisions made throughout the research process [50].

Furthermore, trustworthiness of the research findings were improved by using Creswell's [28] concurrent triangulation strategy. Through mixing qualitative and quantitative data, the aim of the study was achieved in that a deeper understanding of the process of adjusting to diabetes management behaviours of young adults living with type 1 diabetes were formed [51].

\section{Results}

This study was conducted to gain a better understanding of how developmentally appropriate challenges and psychological health variables influence the diabetes self-management behaviours of young adults. Diabetes self-management behaviours were investigated via the personal model created of diabetes, and how social support received by friends and family crystallised through the participants' recollection of experiences and perceptions. Relevant vignettes from interviews and questionnaires were offered to support the concepts. Statement pieces used by participants that preferred to be interviewed in Afrikaans, were translated into English for the purpose of this discussion.

Participants reported the journey towards adulthood were more engaging for the young adult living with type 1 


\section{Psychology \& Psychological Research International Journal}

diabetes opposed to individuals that only needed to adapt to normal developmental stage challenges, asYA3 (female, 19 years of age, Cape Malayan, 6 years since diagnosis) explained: "being diagnosed as type 1 was traumatic ... everything needed changing in my diet and lifestyle ... and I don't wanna die of this thing". In this study, as with others $[9,11,12]$. It was found that young adults were navigating various challenges on their path towards adulthood including the initial diagnosis which could be experienced as traumatic, the commencement with the diabetes care plan as well as the educational sessions and finally, the reality of developing diabetes-related complications.

Participants reported experiences that centered on the age-appropriate challenges of establishing their own identity, autonomy, and effective self-management. Participants' personal identity was not equal to the condition, YA2 (male, 19 years of age, European, 6 years since diagnosis) said, "I am not just diabetes". Participants were very aware of the impact that living with diabetes had on their lives, especially regarding their autonomy and self-management as YA6 (male, 24 years of age, European, 10 years since diagnosis) realized, "I had to take responsibility for myself". In this study, as with others [52] it was found that young adults were navigating various challenges on their path towards adulthood including assuming increased responsibility for their diabetes self-management.

The participants in this study maintained an average HbA1c level of $7.3 \%$ ( $45 \mathrm{mmol} / \mathrm{mol}$ ), which could be categorised in the near-normal range for blood glucose levels. Achieving these levels were seen as part of their responsibility as, "that is where it's supposed to be" (YA3, female, 19 years of age, Cape Malayan, 6 years since diagnosis). Achieving blood glucose levels within the near normal range also filled them with a sense of pride as YA1 (female, 20 years of age, European, 2 years since diagnosis) commented, "you are feeling pretty proud when your HbA1c is under seven". Feelings of control and personal mastery over the diabetes care plan, as well as correct interpretation of their diabetes symptoms indicated enhanced self-management behaviours, according to the study done by Keogh et al. [53]. Thus, identity was influenced through diabetes management behaviours; whenever they interpreted it correctly, it could be seen as positive interaction, which led to feelings of mastery and control. These results corresponded with the IPQ's personal control subscale where $60 \%$ of the participants indicated that they believed in their own ability to manage the condition and take responsibility for their self-management behaviours, as YA6 (male, 24 years of age, European, 10 years since diagnosis) said, "the more I understand it [diabetes], the more I can tweak my lifestyle". This finding was in accordance with findings reported by other researchers $[20,53,54]$ who argued that the score on the IPQ-R's personal control sub-scale and blood glucose control levels of an individual were significantly positively related.

Participants were clear that diabetes was an unpredictable, lifelong experiment and that they have to take responsibility for the management thereof. Participants experienced the diabetes management behaviours as their new reality and, "just something I had to do" (YA3, female, 19 years of age, Cape Malayan, 6 years since diagnosis). In spite of continual progression in the understanding of self-management behaviours, the participants still experienced the unpredictability of the condition, as YA1 (female, 20 years of age, European, 2 years since diagnosis) said, "no day is the same" and "you take it one day at a time" (YA2, male, 19 years of age, European, 6 years since diagnosis). Previous studies [27] found that young adults experienced diabetes management behaviours as being a never-ending experiment and it was undeniably also experienced as such by participants in this study, "I think I'm still trying to figure it out" (YA1, female, 20 years of age, European, 2 years since diagnosis). The quantitative data supported that the participants believed their diabetes to be unpredictable, $60 \%$ indicated higher scores on the cyclical and timeline subscales of the IPQ-R. These results were expected as diabetes is a lifelong, unpredictable condition, as was concluded by various previous studies $[54,55,56$, 57].

Despite maintaining near-normal blood glucose levels, the findings of this study strengthened arguments that young adults were poor at adhering to the diabetes treatment plan, as YA1 (female, 20 years of age, European, 2 years since diagnosis) remarked, "of course there will be times where I say screw it". This inclination to poor adherence was shown by results on the DSMP-SR, where $80 \%$ of the participants obtained low scores, which in turn, were indicative of poor treatment adherence. In addition, all participants scored very low on the IPQ-R's treatment control subscale, which indicated that they believed that their diabetes treatment plan and accompanied self-management behaviours would not control the condition. This could be resultant of a generic diabetes treatment plan that was structured without the incorporation of individual needs and experiences, hence poorer adherence. Previous research concluded that the treatment control subscale gave an indication of the 


\section{Psychology \& Psychological Research International Journal}

adherence ability of the individual - higher scores on the treatment control subscale would transpire in better adherence and glucose control $[20,53,58]$.

The discrepancy between the participants' beliefs about personal control and treatment control led them to experience internal conflict and emotional distress. The additional responsibility of successfully managing diabetes, contributed to increased negative perceptions of the condition, "there's no reward, nothing" (YA1, female, 20 years of age, European, 2 years since diagnosis), and YA2 (male, 19 years of age, European, 6 years since diagnosis) remarked, "and that's the way you go on". All participants agreed that, "it's a full time job, you don't ever stop thinking about it....no break, and no vacation" (YA1,female, 20 years of age, European, 2 years since diagnosis). Participant YA6 (male, 24 years of age, European, 10 years since diagnosis) stated "if I was hungry I'd sometimes just avoid eating because I didn't want to mess up the glucose" and "I find myself going what if I am doing something wrong now that I'm gonna look back on and regret?" (YA1, female, 20 years of age, European, 2 years since diagnosis). In this study, $60 \%$ of the participants reported experiencing negative emotions in relation to diabetes management behaviours (according to the IPQ-R emotional representation subscale). The experience of negative emotions were also evident in the PHI scores that indicated $60 \%$ of the participants did not experience present-day happiness.

The emotional distress influences adherence to the diabetes care plan. This could be summed up by the words of YA4 (female, 24 years of age, European, 22 years since diagnosis), "even with that [diabetes management] I took chances, experimenting, trying everything and not injecting for it, resulting in me suffering the consequences for always" and "it is so frustrating to know your sugar will be jumping around and you can do nothing" (YA2, male, 19 years of age, European, 6 years since diagnosis) even said, "the management plan doesn't work out all the time, kind of frustrating" (YA3, female, 19 years of age, Cape Malayan, 6 years since diagnosis). Participants especially experienced frustration regarding meal plans and food choices as YA1 (female, 20 years of age, European, 2 years since diagnosis) stated, "my mom would be like, why did you eat that and I'm like but I was low. Then she's like, you should tell me first" and "healthy food choices were not a simple task" (YA2, male, 19 years of age, European, 6 years since diagnosis) as well as "my lifestyle was very healthy but it was still a huge flip, it's so frustrating, annoying" (YA1, female, 20 years of age, European, 2 years since diagnosis).
The elevated levels of distress and frustration pertaining to meal plans were further linked to the participant's experience of support from family and friends, "but no one understands ...it can get like upsetting" (YA1, female, 20 years of age, European, 2 years since diagnosis) and "I was tired and frustrated with my mom constantly taking over" (YA4, female, 24 years of age, European, 22 years since diagnosis). This was supported by the DSSQ, which indicated that majority of the participants felt frustrated with others trying to control their meal plan adherence. In addition, the qualitative and quantitative results contradicted each other with regard to social support. All participants stated that they needed support, "obviously you need support" (YA1, female, 20 years of age, European, 2 years since diagnosis) and "it definitely helped a lot" (YA6, male, 24 years of age, European, 10 years since diagnosis), while the results from the DSSQ reflected that $100 \%$ of participants experienced low levels of support from their families and $60 \%$ reported low support levels from their friends. These findings contrasted research that found good social support correlated positively with effective diabetes management behaviours, which in turn, resulted in blood glucose levels remaining at near-normal levels $[59,60,61,62]$.

Participants also experienced emotional distress regarding the consequences of not adhering to the diabetes treatment plan, as YA6 (male, 24 years of age, European, 10 years since diagnosis) said, "it freaked me out", but a quote from YA3 (female, 19 years of age, Cape Malayan, 6 years since diagnosis) was testament of the distress the young adults experience, "knowing about all the things that can happen if I don't manage it, is a huge thing" as well as "I don't want to go down that route, but I want to enjoy my life" (YA4, female, 24 years of age, European, 22 years since diagnosis). According to the IPQ-R's consequence sub-scale, $80 \%$ of the participants experienced emotional distress connected with the consequences of not adhering to the diabetes treatment plan. This study supported work done by researchers $[20,63,64]$ who argued that young adults were wellknown for their non-adherence behaviours and poor selfmanagement of type 1 diabetes, which will result in serious consequences for the individual.

The following clearly shows the interaction of identity, negative emotions, and meaning. Poorly managed blood glucose levels prevented participants from accomplishing their dreams and aspirations, which led to the experience of disappointment, "I am competitive and I know I'm not performing to what I can" (YA1, female, 20 years of age, 


\section{Psychology \& Psychological Research International Journal}

European, 2 years since diagnosis) and "I want healthy kids and for that I must manage it a certain way" (YA3, female, 19 years of age, Cape Malayan, 6 years since diagnosis). This conflict permeated every facet of their lives, to the point where participants actually felt fearful that their children "will suffer and that scares me" (YA3, female, 19 years of age, Cape Malayan, 6 years since diagnosis). By not being able to execute their expectations for the future, participants reported the experience of internal conflict [27] that manifested in experiences of unhappiness and perceived lower quality of life.

Adhering to a diabetes care plan was driven by diverse motivations such as a longing to be like everyone else, "I want to be the same as everyone else" (YA6, male, 24 years of age, European, 10 years since diagnosis) and "all you want is to experience a normal sort of lifestyle" (YA1, female, 20 years of age, European, 2 years since diagnosis). A 'new' normal was sought after by all participants. Yet, the longing for normality came with an emotional package, as YA3 (female, 19 years of age, Cape Malayan, 6 years since diagnosis) said, "I am like not emotionally strong enough to deal with it (management of diabetes)". This longing experienced by the participants was reflected in the MLQ scores where $58 \%$ of the participants indicated that they were actively searching to find meaning in their lives. Thus, participants were actively searching to make sense of the world as viewed through the diabetes lens. This search incorporated participants' desire to find the value and significance of their lives, as well as the purpose of their lives, "it has become more difficult" (YA3, female, 19 years of age, Cape Malayan, 6 years since diagnosis). The high search-formeaning score could also be linked to the participants' wish for their environment to be structured and predictable amidst the unpredictable nature of diabetes; participants searched to understand their 'new normal' with type 1 diabetes.

\section{Conclusion}

In this study, diabetes management behaviours were described as experiences that confronted the young adults with considerable adjustment difficulties. This study highlighted important relations between variables such as the negative emotions that were the result of the identity challenges and restricted food choices. Despite having to adhere to the complex and ever changing diabetes management requirements, the young adults employed different processes to make sense of their world and their diabetes experiences [65].
It could be argued that the relation between the search for meaning and adjusting to diabetes management resulted in the creation of a new normal way of living with type 1 diabetes. This involved changing the way of understanding one's own identity. Through the process of developing autonomy and responsibility, the young adults found it difficult to uphold a sense of happiness and psychological well-being. This development process resulted in non-adherence to the prescribed diabetes treatment plan. In addition, the young adults spoke of experiencing their diabetes management behaviours as a never ending experiment of trial-and-error. A constant search for meaning, and more specifically, finding the new sense of comprehension, would increase their sense of meaning in life [66].

The research further suggested that the nonadherence behaviours enhanced internal conflict in the young adults resulting in experiences of emotional distress. As evidenced in the study, the participants made conscious and deliberate efforts to cope with the diabetes management behaviours and the distress associated with following the challenging meal plans. Participants had to cope with frustration provoking incidents of social support by renegotiating relations that added to their experiences of emotional distress. Hence, in these situations, social support were not contributing to successful diabetes management behaviours for the young adults living with type 1 diabetes.

It could be argued that diabetes created a state of difference within the mind of the young adult as the envisaged future plans and ideas now seemed unreachable and effective diabetes management behaviours had to be initiated to find a new state of consistency. Participants' yearning for living a "normal" life, a life where they do not need to worry about injections and eating plans, were found to be the motivating factor behind their constant efforts to manage the condition effectively, and this process infused their lives with meaning. Through application and adherence to diabetes management behaviours, with the help of trial and error learning, young adults managed to achieve a good level of diabetes control.

\section{Limitations and recommendations}

The current study was only able to provide a snapshot of young adults living with type 1 diabetes and the processes used to overcome challenges to accommodate the condition and the accompanying management behaviours. This study consisted primarily of women $(n=$ 


\section{Psychology \& Psychological Research International Journal}

4); including more men in additional studies is necessary to better understand adjusting to diabetes management. The small sample may limit the transferability of findings. The majority of citizens in South Africa are African and this study had no representatives from this majority population group. All participants were from a more affluent part in South Africa, reporting near-normal HbA1c; including more people with different backgrounds and $\mathrm{HbA1c}$ levels could produce different narratives and, therefore, different findings. The study also did not discriminate between well-controlled and uncontrolled diabetes, which could have important implications for adjusting to diabetes management.

An implication for practice could be that participants needed their autonomy to be preserved through the process of establishing their identity. Young adults want to be included in the decision making process regarding their diabetes management treatment plan. Shared decision making should result in a diabetes treatment plan that meets the goals of both the physician and the individual. Because this is a shared process, the resultant plan should have been carefully constructed so that the individual is able and willing to execute it - i.e. adhere to it. This is in contrast to compliance where a physician dictates a diabetes treatment plan that the individual has not fully participated in content or design, and therefore, may not be capable or willing to execute it, leading to noncompliance. Interventions are needed that aim to enhance young adults' capability of managing various cognitive and emotional challenges of diabetes and increase their adherence. Further research is warranted to understand the meaning-making processes employed towards psychological well-being of individuals.

\section{References}

1. Simpson AR (2008) MIT Young Adult Development Project.

2. Brown T, Childs B, Funnel M, Haas L, Hosey G, et al. (2009) National standards for diabetes selfmanagement education. Journal of Diabetes Care 32(supp 1): 87-94.

3. National Institure for Health and Care Excellence NICE (2008) Diabetes guidelines, pathways, quality standards and advice.

4. Law GU, Kelly TP, Huey DD, Summerbell C (2002) Self-management and well-being in adolescents with diabetes mellitus: do illness representations play a regulatory role? Journal of Adolescent Health 31(4): 381-385.

5. Griva K, Myers LB, Newman S (2000) Illness perceptions and self-efficacy beliefs in adolescents and young adults with insulin dependent diabetes mellitus. Psychology \& Health 15(6): 733-750.

6. Livneh H, Antonak RF (2005) Psycholsocial adaptation to chronic illness and disability: A primer for counsellors. Journal of counselling and development 83(1): 12-20.

7. Loeb SJ, Penrod J, Falkenstern S, Gueldner SH, Poon LW (2003) Supporting older adults living with multiple chronic conditions. Western Journal of Nursing Research 25(1): 8-29.

8. Moser A, van der Bruggen $H$, Spreeuwenberg C, Widdershoven G (2008) Autonomy through identification: a qualitative study of the process of identification used by people with type 2 diabetes. Journal of Clinical Nursing 17(17b): 209-216.

9. Sparud-Lundin C, Öhrn I, Danielson E (2009) Redefining relationships and identity in young adults with T1D. Journal of Advanced Nursing 66(1): 128138.

10. Wood AM, Johnson J (2016) The Wiley Handbook of Positive Clinical Psychology. Chichester, UK: John Wiley \& Sons Ltd.

11. Chaing JL, Kirkman MS, Laffel LMB, Peters AL (2014) T1D through the life span: A position statement of the American Diabetes Association. Diabetes Care 37(7):2034-2054.

12. Rasmussen B, Ward G, Jenkins A, King SJ, Dunning T (2011) Young adults' management of T1D during life transitions. Journal of Clinical Nursing 20(13-14): 1981-1992.

13. Scholes C, Mandleco B, Roper S, Dearing K, Dyches T, et al. (2013) A qualitative study of young people's perspectives of living with T1D: do perceptions vary by levels of metabolic control? Journal of Advanced Nursing 69(6): 1235-1247.

14. Brierley S, Eiser C, Johnson B, Young V, Heller S (2012) Wording with young adults with T1D: Views of multidisciplinary care team and implications for service delivery. Diabetic Medicine 29(5): 677-681. 


\section{Psychology \& Psychological Research International Journal}

15. Silverstein J, Klingensmith G, Copeland K, Plotnick L, Kaufman F, et al (2005) Care of children and adolescents with T1D: a statement of the American Diabetes Association. Diabetes Care 28(1): 126-212.

16. Wysocki T, Taylor A, Hough B, Linscheid T, Yeates K, et al. (1996) Deviation from developmentally appropriate self-care autonomy: Association with diabetes outcomes. Diabetes Care 19(2): 119-125.

17. American Diabetes Association (ADA) (2009) Standards of medical care in diabetes. Diabetes Care 41(Supp 1): S1-S2.

18. Segal DM, Prof. (2017, October 15). Understanding meaning [E-mail].

19. Brassai L, Piko BF, Steger M (2011) Meaning in life: Is it a protective factor for adolescents' psychological health? International Journal of Behavioral Medicine 18(1): 44-51.

20. Hagger MS, Orbell S (2003) A meta-analytic review of the common-sense model of illness representations. Psychological Health 18(2): 141-184.

21. Leventhal H, Nerenz D, Steele D (1984) Illness representations and coping with health threats. In A. Baum A ,Singer J (Edns.), A handbook of psychology and health, Erlbaum, Hillsdale, NJ, 219-252.

22. Snoek FJ, Skinner CT (2005) Psychology in Diabetes Care ( $2^{\text {nd }}$ edn.). New York, John Wiley \& Sons.

23. Steger M (2012) Making meaning in life. Psychological Inquiry 23(4): 381-385.

24. Chew BH, Shariff-Ghazali S, Fernandez A (2014) Psychological aspects of diabetes care: Effecting behavioural change in patients. World Journal of Diabetes 5(6): 796-808.

25. Abraidio-Lanza AF, Vasquez E, Echverria SE (2004) En lasmanos de Dios [In God's hands]: Religious and other forms of coping among Latinos with arthritis. Journal of Consulting and Clinical Psychology 72(1): 91-102.

26. American Diabetes Association (2015) Stress.

27. Willemse M, Deacon E, Segal D (2018) Life stories managing chronic illness: Young adult's experienceswith type 1 diabetes. Journal of Psychology in Africa 28(3).
28. Creswell JW (2014) Research design: Qualitative, quantitative, and mixed methods approach ( $4^{\text {th }}$ edn.). Thousand Oaks, CA, Sage Publications.

29. Mertens DM (2009) Transformative research and evaluation. New York, Guilford Press.

30. Papadopoulou C, Johnston B, Themessl-Huber M (2016) Decay, transformation, and growth: MeaningMaking processes of patients with acute leukaemia within the first year after diagnosis or relapse. Oncology Nursing Forum 43(2): 73-81.

31. Costa C, Breda Z, Pinho I, Bakas F, Durao M (2016) Performing a thematic analysis: An exploratory study about managers' perceptions on gender equality. The Qualitative Report 21(13): 34-47.

32. Wysocki T, Buckloh LM, Antal H, Lochrie A, Taylor A (2011) Validation of a self-report version of the diabetes self-management profile. Diabetes Care 34(8):1701-1705.

33. Wysocki T, Buckloh LM, Antal H, Lochrie A, Taylor A (2012) Validation of a self-report version of the diabetes self-management profile. Pediatric Diabetes 13(5):438-443.

34. Hervaz G, Vazquez C (2013) Construction and validation of a measure of integrative well-being in seven languages: The Pemberton Happiness Index. Health \& Quality of Life Outcomes 11(66).

35. Moss-Morris R, Weinman J, Petrie KJ, Horne R, Cameron LD, et al. (2002) The Revised Illness Perception Questionnaire (IPQ-R). Psychology and Health 17(1): 1-16.

36. Lau RR, Hartman KA (1983) Common sense representations of common illnesses. Health Psychology 2(2): 167-185.

37. Leventhal H, Nerenz D, Steele D, Hillsdale NJ, Erlbaum (1984) Illness representations and coping with health threats. In: Baum A, Singer J (Eds.), A handbook of psychology and health, pp. 219-252.

38. Steger MF, Frazier P, Oishi S, Kaler M (2006) The Meaning in Life Questionnaire: Assessing the presence of and search for meaning in life. Journal of Counseling Psychology 53(1): 80-93.

39. Steger M, Park CL (2012) The creation of meaning following trauma: Meaning making and trajectories 


\section{Psychology \& Psychological Research International Journal}

of distress and recovery. In R. A. McMackin, T. M. Keane, E. Newman, J. M. Fogler (Edns.), Towards an integrated approach to trauma focused therapy: Placing evidence-based interventions in an expanded psychological context. Washington, DC, American Psychological Association Press.

40. Malik JA, Koot HM (2011) Assessing diabetes support in adolescents: Factor structure of the Modified Diabetes Social Support Questionnaire (M-DSSQFamily). Pediatric Diabetes 12(3 Pt 2): 258-265.

41. Malik JA, Koot HM (2012) Education and psychological issues assessing diabetes support in adolescents: Factor structure of the modified Diabetes Social Support Questionnaire (DSSQFriends). Diabetic Medicine 29: 232-240.

42. Tashakkori A, Teddlie C (2003) Handbook of mixed methods in social and behavioural research, Thousand Oaks, CA, Sage.

43. Leech NL, Onwuegbuzie AJ (2010) Guidelines for conducting and reporting mixed research in the field of counselling and beyond. Journal of Counseling \& Development 88(1): 61-69.

44. Atlas.ti (Version 7) [Computer software]. (2012). Berlin, Germany: Scientific Software Development.

45. Braun V, Clarke V (2006) Using thematic analysis in psychology. Qualitative Research in Psychology 3(2): 77-101.

46. Braun V, Clarke V, Rance N (2014) How to use thematic analysis with interview data (process research). In A. Vossler\& N. Moller (Eds.), The counselling \& psychotherapy research handbook. London: Sage.

47. Leedy PD, Ormrod JE (2005) Practical research: planning and design. Upper Saddle River, N.J: Prentice Hall.

48. Corbin JM, Strauss A (1988) Unending Work and Care. San Francisco, CA: Jossey-Bass.

49. Onwuegbuzie AJ, Corrigan JA (2014) Improving the quality of mixed research reports in the field of human resource development and beyond: A call for rigor as an ethical practice. Human Resource Development Quarterly 25(3): 273-299.
50. Lavelle E, Vuk J, Barber C (2013) twelve tips for getting started using mixed methods in medical education research. Medical Teacher 35(4): 272-276.

51. Nieuwenhuis J (2007) Qualitative research designs and data gathering techniques. In Maree, K. (Eds.). First steps in research. Pretoria, SA: Van Schaik Publishers.

52. Monaghan M, Helgeson VS, Wiebe D (2015) Type 1 diabetes in young adulthood. Current Diabetes Reviews 11(4): 239-250.

53. Keogh KM, Smith SM, White P, Mc Gilloway S, Kelly Al, et al. (2011) Psychological family intervention for poorly controlled type 2 diabetes. The American Journal of Managed Care 17(2): 105-113.

54. Davies MJ, Heller S, Skinner S, Campbell MJ, Carey ME, et al. (2008) Effectiveness of the diabetes education and self-management for ongoing and newly diagnosed (DESMOND) programme for people with newly diagnosed type 2 diabetes: Cluster randomized controlled trial. British Medical Journal, 336: 491495.

55. French DP, Wade AN, Yudkin P, Neil HAW, Kinmonth AL, et al. (2008) Self-monitoring of blood glucose changed non-insulin Type 2 diabetes patients' beliefs about diabetes and self-monitoring in a randomized trial. Diabetes Medicine 25(10): 1218-1228.

56. Glasgow R, Hampson SE, Strycker LA, \& Ruggiero L (1997) Personal model beliefs and socialenvironmental barriers related to diabetes selfmanagement. Diabetes Care, 20(4): 556-561.

57. Mc Sharry J, Moss-Morris R, Kendrick T (2011) Illness perceptions and glycaemic control in diabetes: A systematic review with meta-analysis. Diabetic Medicine, 28(11): 1300-1310.

58. Harvey JN, Lawson VL (2009) The importance of health belief models in determining self-care behaviour in diabetes. Diabetic Medicine 26(1): 5-13.

59. Gallant MP (2003) The influence of social support on chronic illness self-management: A review and directions for research. Health Education \& Behavior 30(2): 170-195.

60. Kaplan RM, Hartwell SL (1987) Differential effects of social support and social network on physiological 
and social outcomes in men and women with type II diabetes mellitus. Health Psychology 6(5): 387-398.

61. Rosland A, Kieffer E, Israel B, Cofield M, Palmisano G, et al. (2008) When is social support important? The association of family support and professional support with specific diabetes self-management behaviours. Journal of General Internal Medicine 23(12): 1992-1999.

62. Schiotz ML, Bogelund M, Almdal T, Jensen BB, Willaing I (2011) Education and psychological aspects social support and self-management behaviour among patients with Type 2 diabetes. Diabetic Medicine 29(5): 654-661.
63. DiMatteo MR (2004) Social support and patient adherence to medical treatment: A meta-analysis. Health Psychology 23(2): 207-218.

64. Rapoff MA (2010) Adherence to pediatric medical regimens (2nd ed.). New York, NY: Springer.

65. Park CL (2010b) Stress, coping, and meaning. In S.Folkman (Ed.), Oxford handbook of stress, health, and coping. New York: Oxford University Press.

66. Park CL (2010a) Making sense of the meaning literature: An integrative review of meaning making and its effects on adjustment to stressful life events. Psychological Bulletin 136(2): 257-301. 